\title{
Status and prospects of the EDELWEISS direct WIMP search
}

\author{
J. Gascon \\ (for the EDELWEISS Collaboration) \\ Institut de Physique Nucléaire de Lyon, \\ Université de Lyon, Université Lyon 1, CNRS/IN2P3, \\ 4 rue E. Fermi 69622 Villeurbanne cedex, France \\ E-mail: gasconeipnl.in2p3.fr
}

\begin{abstract}
EDELWEISS is a direct search for WIMP dark matter using cryogenic heat-and-ionization germanium detectors. We report the final results of the second stage of the experiment, EDELWEISS-II, obtained with an array of ten $400 \mathrm{~g}$ detectors equipped with interleaved electrodes for the rejection of surface events. Limits on the elastic and inelastic cross-sections of spin-independent interactions of WIMPs were derived from a total exposure of $384 \mathrm{~kg} \cdot$ day, obtained following fourteen months of continuous operation at the Laboratoire Souterrain de Modane. We also present the prospects of EDELWEISS-III, which plans to accumulate more than $3000 \mathrm{~kg}$.day of data with fourty new $800 \mathrm{~g}$ detectors. All the surfaces of these new detectors are fully covered with interleaved electrodes, resulting in an increase of fiducial mass and of the rejection capabilities.
\end{abstract}

The 2011 Europhysics Conference on High Energy Physics-HEP 2011,

July 21-27, 2011

Grenoble, Rhône-Alpes France 
The EDELWEISS Collaboration performs direct searches for WIMPs [1] using cryogenic germanium detectors [2] to identify rare events where the collision of these elusive particles induce nuclear recoils with kinetic energy in the ten's of $\mathrm{keV}$ range. In these detectors, kept at a temperature of $18 \mathrm{mK}$, the nuclear recoils induce two signals: an elevation of temperature of a few $\mu \mathrm{K}$, measured with a Ge-NTD heat sensor glued to the surface of the crystal providing a truly calorimetric measure of the total deposited energy, and an ionization signal, collected on electrodes covering all surfaces. The dominant background of events due to $\beta$ and $\gamma$ radioactivity can be reduced by factors exceeding $10^{4}$ by comparing the size of the ionization signal to the recoil energy. For the search, the detector are operated in a radiopure underground environment located in the Laboratoire Souterrain de Modane (LSM) where the 4850 meter-water-equivalent rock cover reduces the cosmic-ray background by six order of magnitude. The collaboration has just concluded a search using an array of ten $400 \mathrm{~g}$ detectors with a novel electrode design ("ID" detectors), and is preparing the deployment of an array of forty $800 \mathrm{~g}$ detectors of a new generation ("FID" detectors).

\section{EDELWEISS-II results}

The experiment EDELWEISS [2] set its most recent limits using an array of ten 400 g germanium cryogenic detectors, installed in the Laboratoire Souterrain de Modane (LSM). The experiment is protected by the $\gamma$-ray background with a $20 \mathrm{~cm}$ lead shield and through a very careful selection of all materials. A clean room surrounds the whole experiment and a class 100 laminar flow with deradonised air $\left(<0.1 \mathrm{~Bq} / \mathrm{m}^{3}\right)$ is used when mounting the detectors. Fast neutrons are a particular background as their scattering in the detector can induce germanium recoil similar to those expected from WIMPs. The fast neutron flux originating from the surrounding rock is moderated through a $50 \mathrm{~cm}$ polyethylene shield surrounding the lead shield. The Monte Carlo simulation of the efficiency of that shield has been tested by comparing the predicted attenuation of a strong AmBe source with the observed yields. Given the measured natural neutron background in the LSM [3], less than 0.1 nuclear are expected in an exposure of $400 \mathrm{~kg} \cdot \mathrm{d}$. A muon veto with a $98 \%$ geometric efficiency surrounds the polyethylene shield, in order to tag neutrons created by muon interactions inside the lead shield. The principle of operation and the performance of the germanium detectors are described in Ref. [4]. The originality of the detector design is the efficient use of concentric electrodes on the flat surfaces of the cylindrical crystal surfaces to tag events occurring near them. This is of particular importance, since electron from $\beta$ and $\gamma$ interactions near the detector surface may suffer from bad charge collection, and thus mimic nuclear recoil interaction. To identify surface events, the electrodes on the flat surfaces are concentric annular interleaved electrodes, with a pitch of $2 \mathrm{~mm}$. In this "InterDigit" electrode design (ID), surface events are tagged by the presence of charge on two electrodes on the same side of the detector, resulting in a measured rejection factor for events due to the surface contamination by ${ }^{210} \mathrm{~Pb}$ of $\sim 10^{5}$ [4]. The cylindrical sides of the detectors are fully covered with plain electrodes, operated at lower voltages. No surface rejection can be done in the corresponding volume. Events with charge on these electrodes are therefore rejected. With the additional cut on the interleaved electrodes, corresponding to depth down to $1 \mathrm{~mm}$ below the flat surfaces, the resulting fiducial volume per detector is $160 \mathrm{~g}$ [2].

Fourteen months of data recored in low-background conditions have been collected with the ten $400 \mathrm{~g}$ detectors between March 2009 and May 2010 [2]. A common analysis recoil energy 

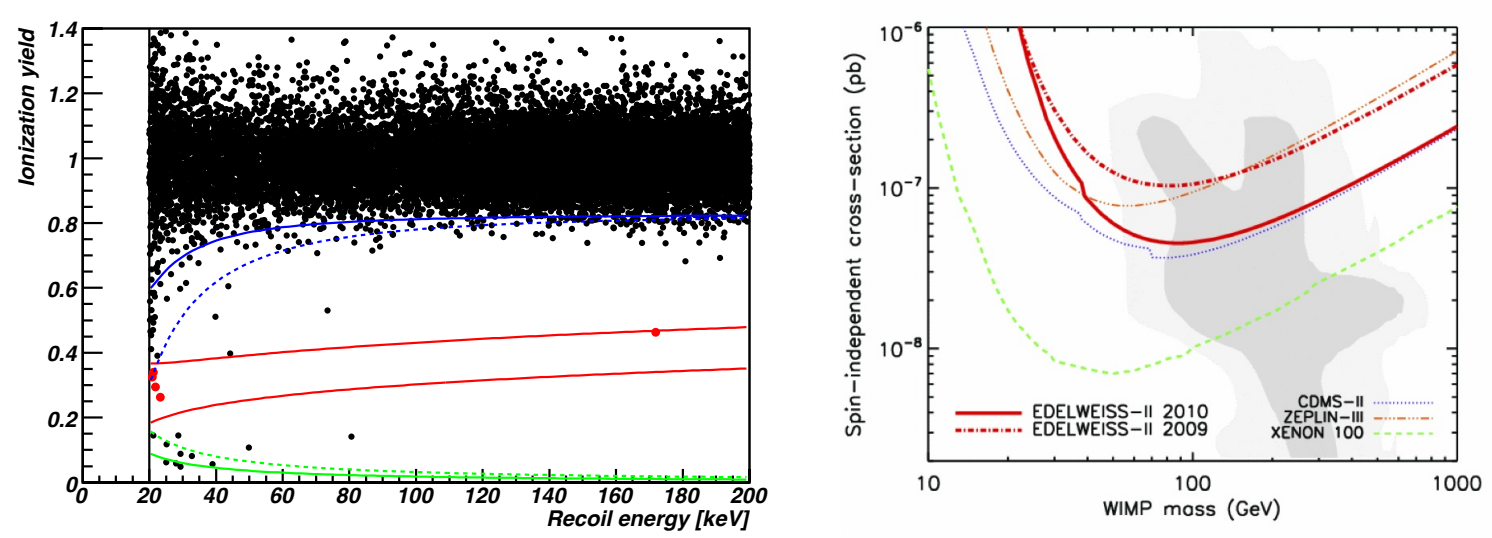

Figure 1: Left pannel: Ionization yield as a function of recoil energy for the $384 \mathrm{~kg} \cdot \mathrm{d}$ EDELWEISS data set. Right pannel: Limits on the cross-section for spin-independent scattering of WIMPs as a function of mass, derived from the present work, together with the limits from CDMS [5], ZEPLIN [6] and XENON100 [7]. The shaded area correspond to the $68 \%$ and $95 \%$ probability regions of the cMSSM scan from Ref. [9].

threshold of $20 \mathrm{keV}$ has been used for all detectors, corresponding to the value at which the efficiency is independent of energy and the rejection of the gamma-ray background deduced from the ionization and heat resolutions is above $99.99 \%$. The total fiducial exposure for nuclear recoils is $384 \mathrm{~kg} \cdot \mathrm{d}$. The data are shown in the left panel of Fig. 1. Four nuclear recoil candidates are observed between 20.2 and $22.5 \mathrm{keV}$, and additional one is observed at $172 \mathrm{keV}$. The resulting limits are shown in the right pannel of Fig. 1, compared with those of other direct WIMP searches [5, 6, 7]. The data has a significant impact on the total sensitivity obtained when combining them with the results of the other search using cryogenic germanium detectors, CDMS [8]. While this relatively small (1.6 kg fiducial) array achieved a sensitivity comparable to other leading searches, its results were limited by the appearance of background. The predicted background in the $384 \mathrm{~kg} \cdot \mathrm{d}$ exposure is 3 events, the largest uncertainty on this number being associated with the presence of the large low-field region below the guard electrodes, and the possible production of neutron via $(\alpha, n)$ reaction in the volume inside the lead shield [2]. This lead to plans to improve the detector design by removing as much as possible the surfaces covered by conventional flat electrodes, and to provide for an internal neutron polyethylene shield.

\section{EDELWEISS-III status and prospects}

Following these observations, the detector design has been improved and it was found possible to covers the entire surface of the detectors with the interleaved electrode pattern, as shown in the left pannel on Fig. 2. With this new design without a low-field guard region, it was also possible to increase the mass of the detector to $\sim 800 \mathrm{~g}$. With these two modifications, the fiducial mass per detector is nearly $600 \mathrm{~g}$. A second thermal measurement has been added to remove any ambiguity in the interpretation of the thermal signal and improve the resolution of the heat measurement. Tests of these new detectors are under way, with encouraging results. In particular, the $\gamma$-ray rejection performance was found to be improved relative to the ID design. The right pannel of Fig. 2 shows 

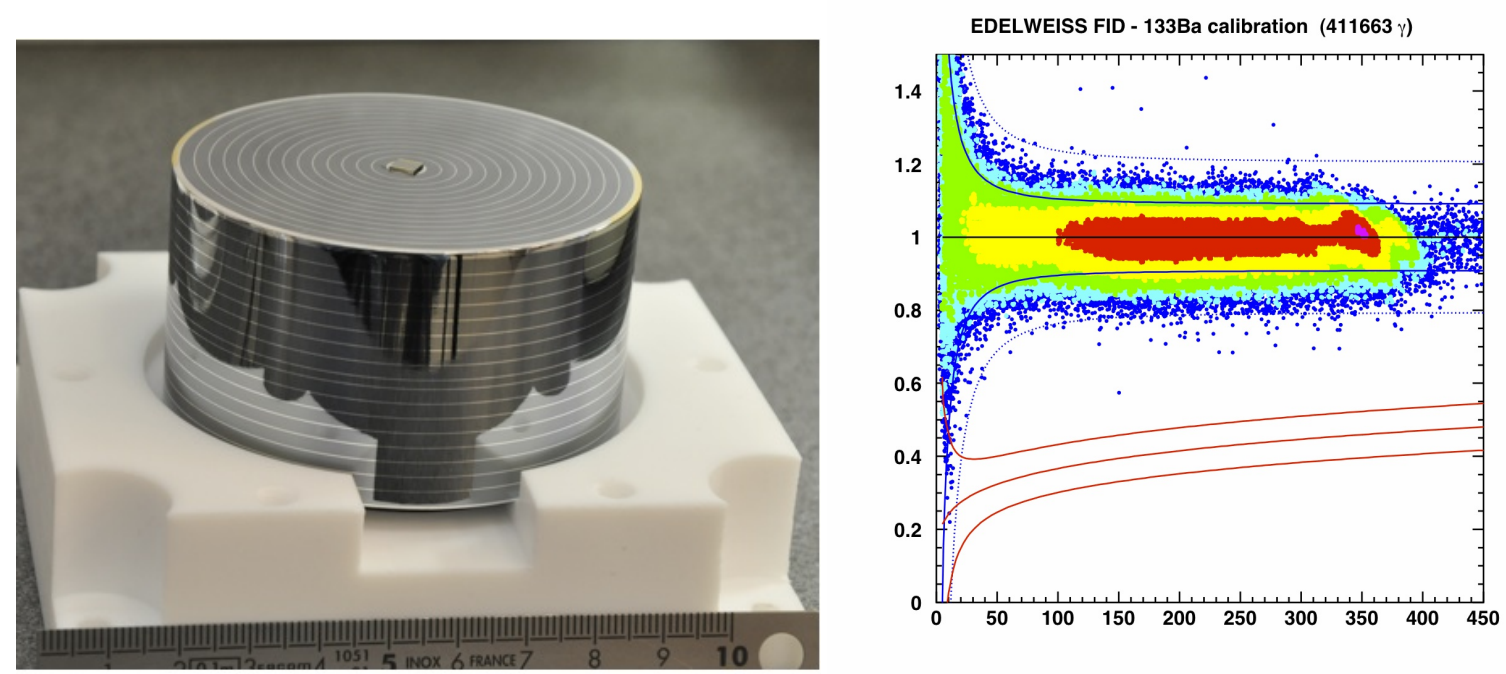

Figure 2: Left: FID detector just after the evaporation of its interleaved electrodes. Right: Ionization yield as a function of recoil energy obtained by exposing FID detectors to more than 400,000 fiducial $\gamma$-ray events.

the results of a $\gamma$-ray calibration of two FID detectors with a ${ }^{133} \mathrm{Ba}$ source. No nuclear recoil candidates are observed after an exposure to more than 400,000 electron recoils.

The construction of an array of forty $800 \mathrm{~g}$ detector, to be completed by 2012, is in progress, with a projected sensitivity of WIMP scattering cross-section of $5 \times 10^{-9} \mathrm{pb}$. This third stage of EDELWEISS will also see its cryogenics and electronics being upgraded for achieving a recoil energy analysis threshold below $15 \mathrm{keV}$. Further developments are being studied in the framework of the EURECA [10] Collaboration, aiming for an experiment in the future extension of the underground laboratory at LSM, with a sensitivity goal of $10^{-11} \mathrm{pb}$. This project brings together the European efforts of EDELWEISS and also of the CRESST and ROSEBUD teams working on heat-and-scintillation detectors, to built a ton-scale multi-target array of cryogenic detectors.

\section{References}

[1] G. Jungman, M. Kamionkowski, and K. Griest, Phys. Rep. 267, 195 (1996); G. Bertone, D. Hooper and J. Silk, Phys. Rep. 405, 279 (2005).

[2] E. Armengaud et al. (EDELWEISS Collaboration), Phys. Lett. B 702 (2011) 329.

[3] R. Lemrani et al., J. Phys. Conf. Ser. 39145 (2006) 145.

[4] A. Broniatowski et al. (EDELWEISS Collaboration), PLB 681 (2009) 305.

[5] Z. Ahmed et al., Science 327 (2010) 1619.

[6] V.N. Lebedenko et al., Phys. Rev. D 80 (2009) 052010.

[7] E. Aprile et al. (XENON Collaboration), Phys. Rev. Lett. 107 (2011) 131302.

[8] Z. Ahmed et al. (CDMS and EDELWEISS Coll.), Phys. Review D 84 (2011) 011102.

[9] L. Roszkowski, R. Ruiz de Autri and R. Trotta, JHEP 07 (2007) 075.

[10] H. Kraus et al., J. Phys. Conf. Ser. 39 (2006) 139. 